\title{
A Macintosh II psychophysiology system
}

\author{
TIMOTHY C. BATES \\ University of Auckland, Auckland, New Zealand
}

\begin{abstract}
An integrated data-acquisition and -analysis system for doing psychophysiology on Macintosh II computers is described. Plug-in cards allow the system to meet the technical demands of psychophysiological research-including 24-channel, 12-bit resolution and sampling of scalp potentials taken at a sampling rate of $400 \mathrm{~Hz}$. Use of a compiled graphical data-flow language to program this equipment brings the implementation of sophisticated methods within the reach of many experimenters. Some applications of this system to multichannel evoked potential research are described, including P50 conditioned-disinhibition and augmenting/reducing experiments. Extensions of the system into such areas as topographical brain mapping are also noted.
\end{abstract}

We have recently developed a computer-based system for doing human psychophysiological research. We required a flexible system that would provide digital control of stimulus generation simultaneously with digitization of physiological responses, including evoked brain potentials (EP), EEG, muscle tension, and heart rate. We wanted a system with sufficient spatial and temporal resolution to allow topographic mapping of EPs, a procedure in which a "map" of the brain is rendered in colors that portray electrical activity as it is distributed across the recording sites. To achieve this goal, we assembled a system able to record from up to 32 channels, at sample rates exceeding $500 \mathrm{~Hz}$ and with a precision of 12 bits (meaning that samples can take any of 4,096 discrete levels). A high priority was that the system be programmable as nearly as possible in terms of the abstract experimental block-diagram understood by researchers. This was in distinction to the traditional process, which requires translation from the mental picture of the block-diagram into languages such as $\mathrm{C}$ or Pascal-languages that are oriented more toward the capabilities and needs of computer hardware than they are to the structures of experimental design. Reasonable cost was a third, important, consideration. The system schematic is shown in Figure 1.

\section{HARDWARE FOR DATA ACQUISITION AND STIMULUS CONTROL}

\section{Amplifier and Interfacing Problems}

First we chose an amplifier system. We chose to purchase a commercial EEG rather than build our own machine. ${ }^{1}$ Although some costs may be saved with inhouse machines, we felt safer with the manufacturer's technical support and ensured safety. It was important that the amplifier parameters and montage be controllable by

The author would like to thank all the anonymous reviewers of this paper for their helpful and constructive comments. He also sincerely appreciates the support of Dr. G. Mangan and Philip Morris, Inc. during this project. Correspondence may be addressed to Timothy $\mathrm{C}$. Bates, Department of Psychology, University of Auckland, Private Bag, Auckland, New Zealand. an external computer and that the outputs be at a suitable voltage for subsequent digitization. We therefore chose the 21-channel Nicolet EEG IA98. This machine has a built-in RS-232 interface, which provides a low-speed serial data link that allows computer control of the filter settings, amplification, and montage of the EEG machine. Analog voltage outputs with a range of $\pm 1.4 \mathrm{~V}$ (the IRIG or tape-recorder standard level) provide a signal appropriate for analog-to-digital (A/D) conversion. Alternative machines of equal quality and utility are available from manufacturers such as Nihon Kohden. Further options worth consideration are the "bread box" amplifier sets designed for use in computer-based systems. Because they do not provide a paper trace output, these sets are cheaper than traditional EEG machines are. Nicolet manufactures a 32-channel computer-controlled model that sells at a price comparable to that of their 21-channel EEG machine.

Although most modern EEG machines have outputs suitable for digitization and may also include a built-in control system such as an RS-232 interface, gaining control of older but still useful devices such as photic and auditory stimulators often presents an intransigent obstacle to automating a laboratory. Usually these devices have a transistor-transistor level or TTL input that allows an external signal of $5 \mathrm{~V}$ to trigger the device. Often, however, other device settings are controlled by analog knobs or switches, for which digital controls are not provided.

Given the need in nearly all modern paradigms to change stimulator settings between subsequent stimulus presentations, it is essential to provide digital control of stimulus parameters. Our solution to this problem involved two strategies. The first was to avoid this older equipment whenever possible, by using a computer monitor and speaker to generate stimuli of arbitrary complexity under program control. When this was not possible, we used existing equipment and bypassed all mechanical switches with switches controllable by digital outputs from a computer. To take the instance of a rotary control with four positions, a one-to-four multiplexer chip can be used 


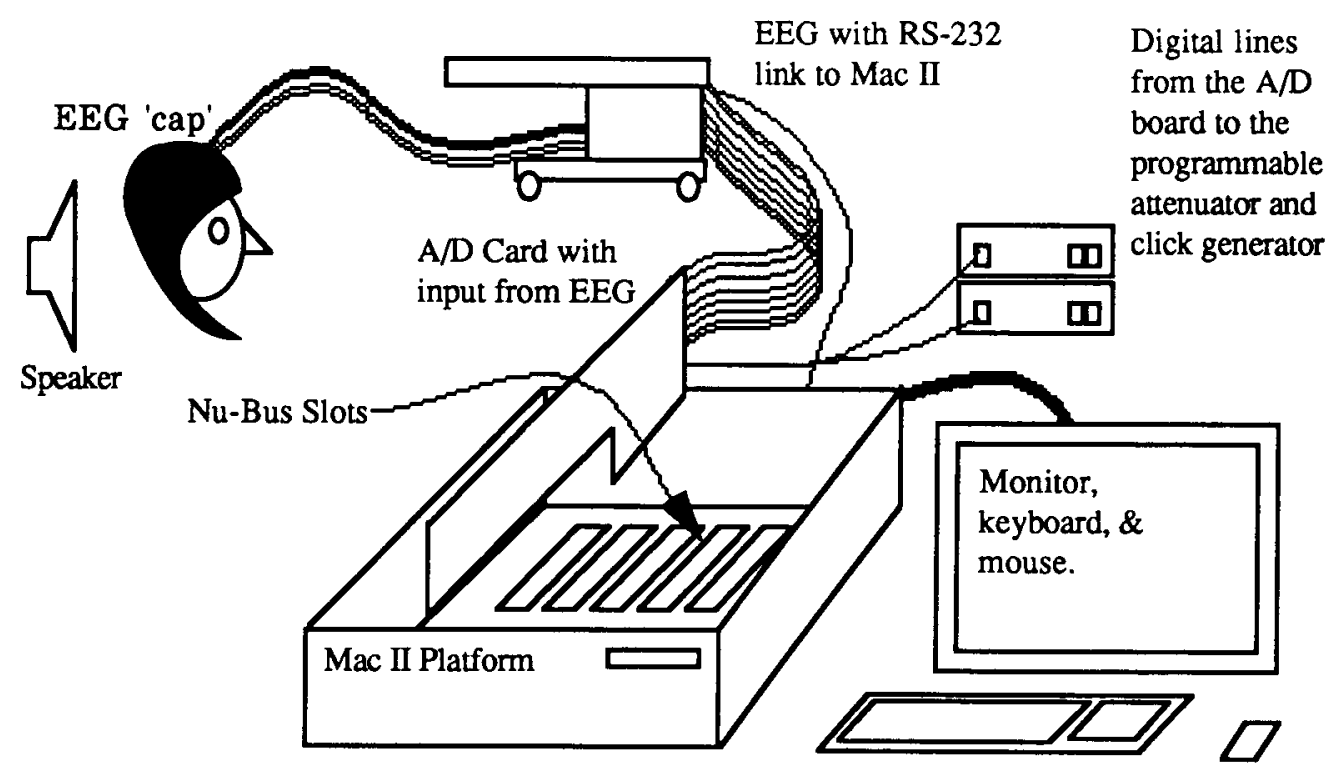

Figure 1. Schematic outline of the system. Shown are the cap used to secure recording electrodes; the EEG machine, which amplifies the EEG according to computer controlled settings; NuBus card(s) to digitize EEG; and the RS232 and digital $\mathrm{I} / \mathrm{O}$ lines used to control and trigger external equipment, such as the click generator and speaker. The whole system is controlled with the Macintosh monitor, keyboard, and mouse.

to replace the function of such a control. With this chip, the states of the control lines from two digital inputs are decoded into a switch setting. Some variation will be necessary, depending on the exact problem. For instance, if the control is carrying substantial voltage or current, this must be switched through a control transistor, or if one wishes to switch an analog signal, a chip allowing analog signal switching must be selected.

\section{Computer and Data-Acquisition-Card Choices}

For a general-purpose microcomputer to integrate this equipment into a data-acquisition system, it must provide a bus or backplane into which data-acquisition cards can be installed. In addition, a high-speed route for data to be stored into memory-preferably a direct memory access (DMA) system - as well as suitable data-acquisition cards must be available. The Apple Macintosh II platform, when equipped with NuBus boards from National Instruments (Austin, TX), conforms to this specification. ${ }^{2}$ The Macintosh NuBus provides a sophisticated system backplane that allows installed cards to arbitrate for control of the total system. NuBus does not, however, implement DMA - a prerequisite for very-high-speed data transfer. This DMA requirement was met with the NB-DMA-2800 board from National Instruments, which provides block and burst mode DMA as well as a 1-MB/sec interface for external instruments with a GPIB interface. In order to digitize the analog EEG signals, we used two NB-MIO16-9 boards. Each of these boards digitizes 16 channels of data at rates up to $100 \mathrm{kHz}$ with 12-bit accuracy. In addition, they each provide three 16-bit programmable clocks, two channels of 12-bit analog output, and eight digital I/O lines. These boards are interconnected by a RTSI bus, which provides real-time system-wide integration through a software-switchable path for connecting timing signals and data between boards with submicrosecond accuracy. An instance of the use of the RTSI bus is the provision of a high-speed path for data between the $A / D$ and DMA boards. Because of this high level of integration, the programmer is able to synchronize the execution of independent tasks such as stimulus control and acquisition of data across independent channels. This is essential in any application that relies on response averaging or on the measurement of response latencies.

By using two NB-MIO-16-9 boards, we achieved a useful sampling rate ranging from $100-\mathrm{kHz}$ sampling across two channels down to $500 \mathrm{~Hz}$ with 32 channels. This sample rate anomaly exists because the boards do not implement sample-and-hold circuitry, and so a skew (equal to the time required to make a $D / A$ conversion multiplied by the number of channels sampled on each board, $160 \mu \mathrm{S}$ in the worst case for the NB-MIO-16-9) exists between samples from each channel, which may, at high sample rates, become a considerable proportion of the sampling period. This is important if information on interchannel coherence is important, as it inevitably is in such applications as topographic mapping, for instance. However, at typical EEG bandwidths of less than $100 \mathrm{~Hz}$, this "smearing" effect is negligible. Should higher bandwidths be desired, one is forced to use fewer than 32 channels. As it stands, most EP research can be achieved with sampling rates of less than $400 \mathrm{~Hz}$ (allowing meaningful anal- 
ysis of signals up to $200-\mathrm{Hz}$ bandwidth according to Shannon's sampling theorem). For researchers requiring more advanced data acquisition, boards are available to digitize data at higher accuracy (16-bit) or faster speeds $(1,000,000$ samples/sec $)$.

A further limitation of the NB-MIO-16-9 is that its sample rate is specified by only one clock. To achieve a quick scan of all channels, followed by a relatively long delay of several milliseconds before the next scan-thus achieving the desired goal of pseudosimultaneous acquisition of data at an overall sample rate of $200-500 \mathrm{~Hz}$-we had to write software that programmed two clocks, so that one generated pulses at the desired per-channel sampling rate and the second clock oscillated at the between-channel rate but was gated by the first. The output of this second clock caused the quick scan to occur when it received the first clock's pulse. Newer boards allow one to simply specify a scan rate and an overall sample rate.

\section{SOFTWARE: THE CHOICE OF A PROGRAMMING LANGUAGE}

Although perhaps a few micro- and minicomputer systems now provide these levels of hardware performance, the biggest hindrance to the routine use of computers in the laboratory has in the past been software. To set up complex experiments in a quick and flexible fashion while attempting to retain direct control of the implementation process has often been a frustrating endeavor. Recently developed visual environments for icon-based data-flow programming have made complex program structures much easier to visualize and to program.

To describe the system for doing psychophysiology developed here, it is necessary to define the concepts involved in these new languages before giving a concrete example of their application in psychophysiological research.

Visual programming is well described by Shu as "the use of meaningful graphic representations in the process of programming" (Shu, 1988, p. 9). A data-flow program may be defined as one that is "translatable into a graph whose nodes represent functions and whose arcs represent data dependencies between functions" (Agerwala \& Arvind, 1982). These two concepts of programming are implemented on the Macintosh in at least two languages: Prograph 2.0 (GKS Systems, Halifax, Nova Scotia) and LabVIEW 2.1 (National Instruments, Austin, TX). Prograph is an object-oriented language that provides an extensive "tool kit" of reusable and extensible software for quickly building Macintosh applications. It is not, however, designed specifically for scientific work. LabVIEW, although it does not implement the full suite of object-oriented features, has more powerful features for psychophysiological experimentation. Because of this, we will refer to the LabVIEW implementation in the rest of our discussion of these modern languages.

\section{Virtual Instruments, Front Panels, and Diagrams}

LabVIEW represents all system resources (be they physical, such as an EEG machine, or software, such as a power-spectrum algorithm) as virtual instruments (VIs). A VI consists of a front panel, diagram, icon, and connector. The front-panel window emulates the front panel of an equivalent physical instrument through which the user gives commands and receives output (see Figure 2). These front-panel windows are paired with a diagram window in which programs are written using a language called G (see Figure 3). $G$ is the compiled graphical language provided by LabVIEW 2 .

The first thing one notices about $G$ is that it provides a visual programming environment. This term refers to the implementation of all program resources as graphic objects rather than text code. Thus, the function performing an addition and storing the result, coded in $\mathrm{C}$ as $x+=y$; or $z=x+y$, is represented in LabVIEW by the icon shown in Figure 4. This icon provides a visual mnemonic for the function and provides a connector interface for the function's data input and output. The icon

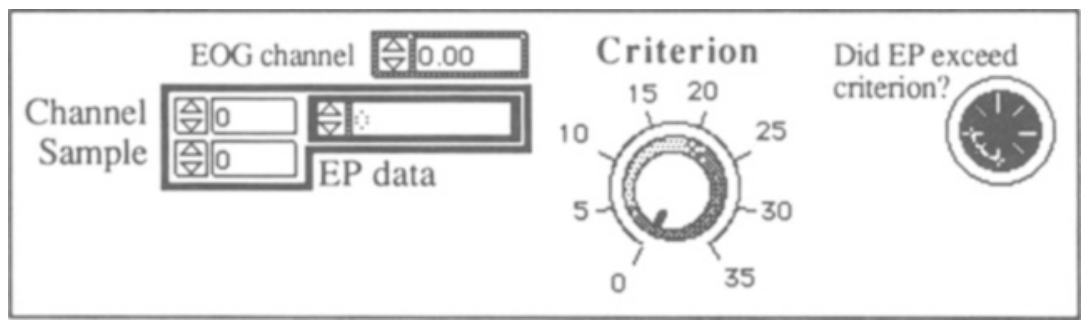

Figure 2. Front panel of a virtual instrument for detecting eye blinks. Front-panel controls and indicators are used here as the variables in an eye-blink detector. Data from one multichannel evoked brain potential trial are input as a two-dimensional array. Individual data points may be examined by putting appropriate values in the two array-index boxes. At present, the index is to the top left of the array (LabVIEW arrays are zero-based). Two numeric values - the channel number to be examined for eye-movement artifact as determined by electrooculogram (EOG), and the maximum tolerable EOG value-are also shown. Note that the criterion input control has been made into a knob for ease of use and understanding. EOG artifact is indicated by a Boolean panel light, shown here set to true. 


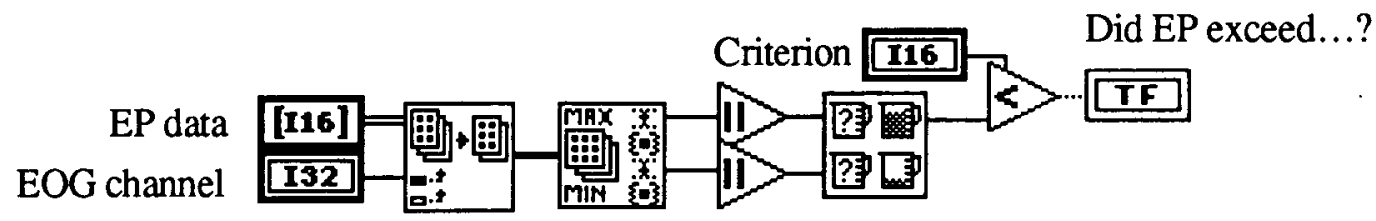

\begin{abstract}
Figure 3. Block diagram of the eye-blink detection virtual instrument (VI) in Figure 2. Reading from left to right, this program diagram shows the evoked brain potential data and electrooculogram (EOG) channel-selector values from Figure 2 flowing into an array-indexer VI that can output a selected slice or element of an array. In this case, the output is the EOG channel. Note the difference in wire type between the two-dimensional input array and the one-dimensional output array. The EOG channel passes into a VI that determines both the maximum and minimum values that the channel takes. These results are then converted to absolute magnitudes, after which a comparison $V 1$ passes on the largest of the two. Finally, the "<" comparison is made between the setting of the criterion control and the EOG excursion. If the specified criterion on the channel is less than the maximum deflection-that is, if the " $<$ " icon output is true, the front panel Boolean is set to true, indicating that the criterion has been exceeded.
\end{abstract}

for the VI in Figures 2 and 3 is shown in Figure 5. A window similar to that in Figure 5 can be called up for all VIs, providing on-line help regarding input types and so forth.

A second distinctive element apparent in Figure 3 is G's embodiment of the data-flow concept of programming. Visual data-flow programming consists of forming a block diagram in which processes are represented by icons and the flow of data through these processes is specified by graphical links.

Using conventional code, one could write a procedural fragment to detect an eye blink roughly as follows:

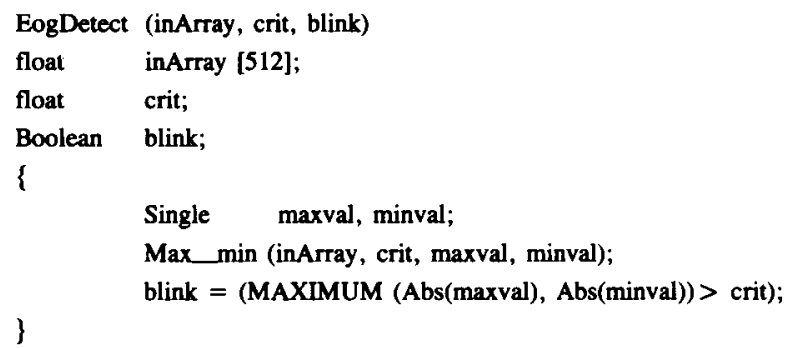

By contrast, the LabVIEW block diagram in Figure 3 outlines the flow of data through a visible "chain" from front-panel controls, through intermediate processes, and out to a front-panel display. The data flow is not restricted to processes as simple as that depicted here. A dataacquisition VI can send data arrays to be transformed

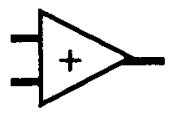

Figure 4. An example icon representing the virtual instrument for addition. Because it is polymorphic, this icon can add any two quantities, be they Boolean, integer, floating-point, or even multidimensional arrays. By the same token, the values to be added need not be of the same type-that is, an integer can be added to a threedimensional array of single precision numbers, the output being the array of floats with the scalar quantity added onto each element. through such supplied icons as filters and spectral analyzers, or through statistical analyses such as nonlinear fitting, built-in mathematical functions such as that which converts a waveform to a cubic spline representation, or even into an external procedure written by the user in C or Pascal. One can store output to disk in most formats simply by using built-in disking icons, or it can be exported to a spreadsheet file, again by using a built-in icon.

A third element of the power of this system rests in G's extensive provisions for hiding or abstracting information. Because LabVIEW is structured (as, e.g., Pascal, with its provision for procedures to call each other by name), VIs can be nested and may call one another (recursive calling of an instrument by itself is not, however, implemented). The visual layering that emerges as a result of structured programming with icons naturally hides details that are irrelevant to each layer and thus presents one with the abstract process being programmed. If one wishes to examine or alter the internal mechanism of any of the iconic modules, a simple mouse click brings one a similarly abstract visual representation of this subfunction.

The provision of a cluster data type allows the formation of what in Pascal are called records (C structs). Graphically, this corresponds to placing several data sources or sinks inside a frame. This complex data type can then be inserted into a superior cluster or an array of clusters, formed simply by placing the cluster into an array holder on the screen. This is useful for grouping an array of EP data together with strings describing the subject's name, sites recorded, and so forth. A common use of the cluster is to bundle together several arrays of data for display in a multiplot graph such as that shown in Figure 6.

The fourth benefit of LabVIEW is an emergent property of the language. Multiple data flows can be programmed, and they will automatically execute in parallel (if possible) processes firing as soon as all their inputs are available. Parallelism conveys two direct benefits. First, the programmer no longer has to arbitrarily decide on the order in which code will be executed when this order is irrelevant to the task. LabVIEW has a multitasking ker- 


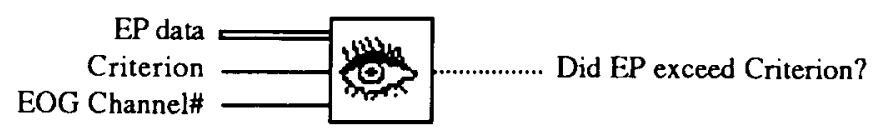

EOG detect

Figure 5. The icon of a virtual instrument (VI) for detecting eye blinks. Seen here is the icon of the VI shown in Figure 3. The small picture calls to mind the purpose of the VI, and the labels on each input describe the input and indicate the appropriate region for wiring onto it. Each input type is denoted by the graphic form of its input wire; the Boolean output, for instance, is signified by a dotted line.

nel that executes VIs in parallel where possible, according to round-robin allocation of processor time. By escaping from the single-channel von Neumann computer model, which executes code in a predefined single sequence, the programmer using LabVIEW is able to think much more closely in terms of the process being achieved than the fashion in which the computer achieves it. An additional interesting feature that may be relevant in future computing environments is that the data-contingency information necessary for compiling efficient parallel- processor code is intrinsic to the program-that is, not only is the illusory sequence of events laid down in older sequential languages not specified, but the independence of processes in the program is specified implicitly. One can imagine a compiler written to devolve tasks onto independent processors if these should be detected.

\section{Control Structures in $\mathbf{G}$}

Related to the parallel nature of data-flow programming mentioned above are the control structures provided by

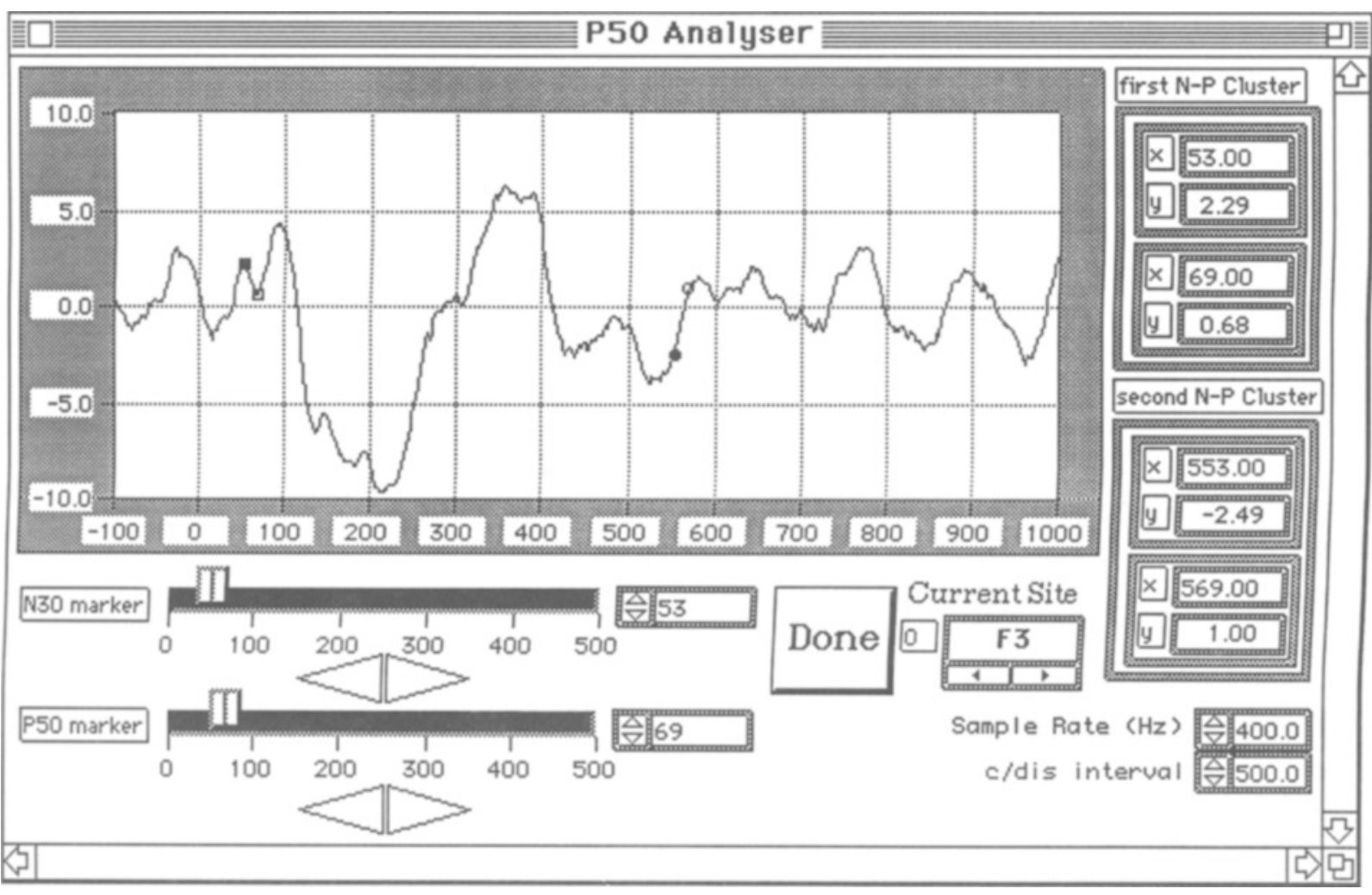

Figure 6. P50 analyzer front panel. The panel is displaying one channel of auditory evoked brain potential recorded in response to two clicks, one presented at $0-\mathrm{msec}$ and the second at $500-\mathrm{msec}$ latency. The identity of the channel is indicated by the ring control at the lower right. On the graph are also plotted four markers, which are controlled in pairs by the two horizontal slides immediately below. The members of each pair of marker points are spaced 500 msec apart and are set by clicking at a point on the slide, using the triangular scroll cursors, or for more precise control, by direct typing of desired latency values into the control. The VI outputs the latency and amplitude of $\mathbf{N 3 0}$ and P50 for each of the two stimuli. 
LabVIEW. Often in psychophysiology, processes that have independent data requirements must nevertheless execute in a certain order; stimulus presentation must be related temporally to data acquisition, for instance. To control the execution order of code, to allow branching to different code depending on test flags, and to allow the repetition of code, LabVIEW provides for- and whileloops-a case structure and a sequence structure.

The for-loop structure has automatic indexing. Rather than require explicit definition of the number of times that the array will iterate, a LabVIEW for-loop simply executes once for each element of an array wired to it as an indexed input. One can of course specify $n$ explicitly, whenever this is required or desired. The while-loop operates conventionally, as does the case structure. The sequence structure does not have a direct equivalent in other languages, because it exists to help direct the flow of execution. For extended sequential operations, a graphical sequence can be programmed as a stack of "film" frames similar to a cartoon animation sequence. Execution order can also be directed by wiring the output of a previous VI to a dummy input terminal on a subsequent VI. The second VI will wait until it receives the dummy input, thus ensuring sequential execution.

\section{Data Types and Primitive Functions in $\mathbf{G}$}

Because the flow of data is determined by graphical connections between processes, the need for variable declarations is eliminated; variables are created, copied, and destroyed automatically to meet the needs of programmed wiring. Another problem with conventional languages involves specifying and remembering data types. In a visual environment, if two points can be connected, they are type-compatible. Three features resolve most type conflicts. The first aid is that in LabVIEW, data types are graphical, so that one is visually reminded of a datum's type at all times. Second, just as in C, automatic and userspecified type coercion can often change inputs into the form required by their receiver-that is, an extended precision real can be converted to an integer. LabVIEW also uses a feature borrowed from object-oriented programming: polymorphism. A single LabVIEW primitive can respond correctly to most possible input types. For example, the addition icon shown in Figure 4 is able to add inputs of any type, be they mixtures of integers, reals of any given precision, or even multidimensional arrays of these basic types. If a single integer and an array of reals are input to the addition function, the function will convert the integer to the higher precision format and output the array of reals with the integer value added to each element.

A great benefit for the experimenter is the very large library of primitives and included functions that comes with LabVIEW. VIs are provided to handle reading and writing to disk files and GPIB or RS-232 interfaces at a very high level. Front-panel objects exist to display multiplot graphs and strip charts. In terms of signal generation or analysis, VIs are provided for the production, anal- ysis, and windowing in both the time and frequency domains of arbitrary signals. Some examples of the included functions are the fast Fourier transform and autocorrelation functions, along with variants of the Fourier process such as Hartley and Hilbert transforms. A power function VI is provided, as are several kinds of finite and infinite response filters for high-, low-, bandpass, and bandstop filtering. An extensive suite of mathematical functions is also included.

\section{EXAMPLE PROJECTS}

Our first project was a 21-channel auditory EP experiment implementing the P50 conditioned-disinhibition paradigm developed by Freedmans' group (Adler et al., 1982), in which the stimulus consists of paired auditory clicks with a 500 -msec interval between members of a pair and a $10-\mathrm{sec}$ interval between presentations of the paired stimuli. The brain electrical responses to several such trials are averaged, and positive waves at a latency of $50 \mathrm{msec}$ (hence the nomenclature P50) from the first and second clicks are detected-the variable computed being the amplitude ratio of these two responses. Failure to inhibit responding to the second pulse is implicated in vulnerability for schizophrenia (Adler et al., 1982).

Twenty-one Sn electrodes were located at chosen scalp sites, using a custom ECI Electro-Cap (Electro-Cap International, Dallas, TX), and the response at each site to each stimulus pair was recorded for $1,000 \mathrm{msec}$, beginning $100 \mathrm{msec}$ before the first click in each stimulus pair. Sampling was done at $440 \mathrm{~Hz}$, using two NB-MIO-16-9 boards, one sampling 16 and the other 8 channels, with 3 channels not connected to the EEG signal because the boards can only scan numbers of channels that are a power of two. A master pulse from one of the NB-MIO-16-9's programmable clocks started each trial. This pulse initiated the programmed $A / D$ conversion sequence on each board and started a delay clock, the output pulse of which triggered the click generator when the baseline time had elapsed. On-line artifact detection consisted of monitoring the F7 response (a site close to the left eye) and determining whether it had exceeded a criterion microvolt level set at a front-panel control; if the channel was clear of artifact, an appropriate case structure added the multichannel EP array into a running store. This sequence of events was programmed as a virtual instrument and nested in a while-loop, which incremented a trial number variable each time a successful trial executed.

Recording continued until 42 artifact-free trials had been acquired. During this time, 14-trial averages of the vertex electrode $(\mathrm{Cz})$ response were graphed on screen to allow the operator to detect any abnormal patterns of activity. The program has since been modified to show the response to each trial as well as a cumulative average. At the conclusion of recording, a grand average for each channel is immediately plotted on screen, along with the two-dimensional numerical array representing the EPs at each site for each sample. 
The P50 data were archived with LabVIEW's integrated database facility. This built-in data logger allows all frontpanel variables, experimental control settings, subject and experiment variables, and a date mark to be stored on disk in a single record that is retrievable either manually or by program. With program retrieval, an analysis VI can recall data from an acquisition VI without user intervention. This is described below.

To analyze the P50 data, a program was written to recall stored runs and present the EP data on screen, thus allowing the P50 to be measured graphically. The P50 amplitudes at each channel along with all the original experimental data were then recorded as a cluster in the analysis VI.

Detection of the P50 is difficult to automate entirely, because it often rides on top of a much larger negative potential (the N100) and varies in shape between recording sites. In order to remain sensitive to these differences yet retain as much automatic processing as possible, a program was written to display the EP for one channel along with four markers, which can be scrolled to coincide with the peak and trough points of the two P50 waves (see Figure 6). Two of the markers are slaves that simply lag the first markers by the 500-msec interstimulus interval. A stable P50 can be analyzed very quickly, and the experimenter can still verify the more difficult cases. Once this analysis is complete, the stored data are written to a text file complete with subjects' names and channel identifiers for MANOVA analysis in SuperANOVA (Abacus concepts).

Our next application extended the P50 instrument to the augmenting-reducing paradigm (Buchsbaum \& Silverman, 1968; Buchsbaum, Haier, \& Johnson, 1983). In this paradigm, separate averages are obtained for each of a range of stimulus intensities, the relevant variable being the slope of the function relating stimulus intensity to the amplitude of an EP wave-for auditory stimuli, the N1-P2 wave amplitude. We used an auditory click stimulus at 10 distinct intensities, presented in three blocks of 20 trials with the blocks arranged in a random order. To achieve this, we inserted the basic EP module developed for P50 into a for-loop that delivered one block on each cycle and accumulated the recorded EPs into a three-dimensional array containing data from each channel for each intensity of stimulus at each time sample. As a check on data integrity, the EP to each block was graphed, and at the end of the experimental session, a graph of the response at one channel for all intensities was produced. The frontpanel data were again logged for analysis.

Analysis of the $A / R$ data consisted of passing the acquired data through a lowpass fourth-order Butterworth filter and then determining the maximum points within two windows defining the boundary of the N1 and P2 waves, respectively. A fragment of the VI written to accomplish this is shown in Figure 7. The resulting data were written to text file, and a MANOVA was carried out in which each channel was used as a dependent variable and intensity was a repeated measure. This was again done with SuperANOVA.

Working from the basic multichannel data-acquisition VI, we have implemented several other paradigms by adding VIs to control stimulators and control structures, in order to direct the order of stimulation and acquisition events. One example of this work was the writing of multichannel EEG to disk files while the frequency of photic

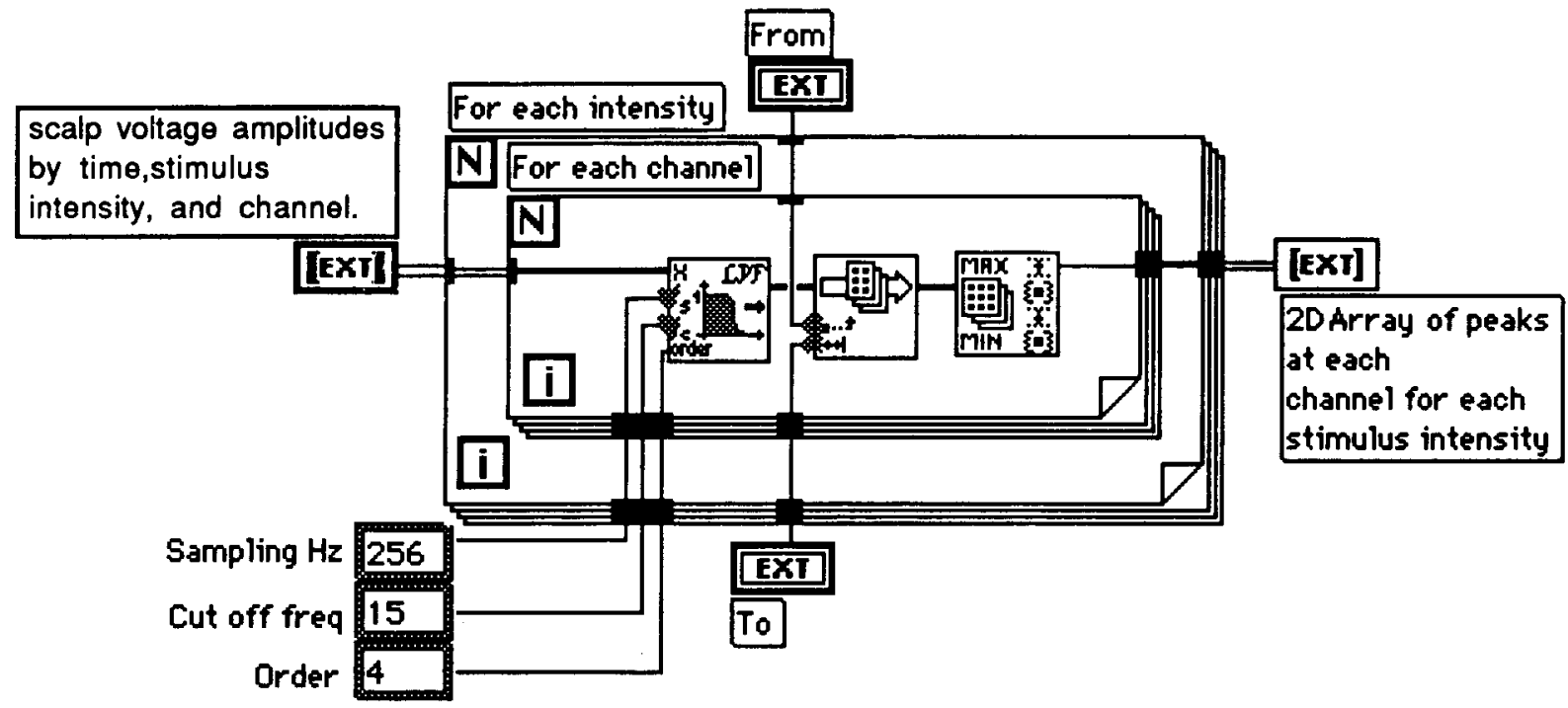

Figure 7. Code fragment from the $\mathbf{A} / \mathbf{R}$ analyser. In this fragment, a three-dimensional array holding the evoked brain potentials (EPs) recorded from 21 channels at each of 10 stimulus levels is indexed by two autoindexing for-loops. In this manner, the first for-loop operates once for each stimulus intensity, and the second loops through single channels of data until all 21 recorded at that intensity have been analyzed. Each EP is passed through a lowpass filter, and the maximum value is determined within a subset of the EP defined as the peak window for the wave in question. The results of each of these determinations are accumulated on the for-loop boundaries, resulting in a two-dimensional array that stores the wave peak value for each channel at each intensity. Note the array types, indicated by wire thickness and shading. 
driving stimuli was varied. Data analysis has also been varied; the EEG data mentioned above, for instance, were analyzed with the built-in filtering and power-spectral, correlation, and array-manipulation VIs.

\section{EXTENSIONS TO THE SYSTEM}

The system outlined above is complete in itself for the acquisition and analysis of data. It may of course need to be extended for specific work. An example of this was provided when we used a form of interapplication communication (IAC) to implement a flexible color topographic mapping system.

Analyses of multichannel recordings can be facilitated if the data recorded from each scalp electrode are mapped onto an outline of the head, using color to represent quantitative values while displaying the topography of brain activity at any given slice of the time or frequency domain under study. LabVIEW 2.0 supports code interfaces for binding external code into LabVIEW VIs, but for applications as substantial as mapping, it may be more practical to write an independent program. If readers need to build such specific applications, an excellent resource is the book Macintosh Programming Primer (Mark \& Reed, 1989 ) in combination with the Think C program (Symantec Corp., 10201 Torre Ave., Cupertino, CA 95014) and Apple's Inside Macintosh, Volumes 1-5.

A major problem encountered in producing topographic maps in real time was to devise a means of achieving highspeed transfer of information out of LabVIEW to a place where it could be shared by another application. Writing the data to disk could not provide speeds suitable for realtime mapping, so a mechanism had to be devised to allow the "piping" of data out of LabVIEW into a memory space accessible to the mapping application. Because the current $(6.0 \times)$ versions of Apple System software do not support interapplication communication, we have emulated this with an INIT (a code resource that is run automatically when the machine is switched on). The INIT reserves memory in the Macintosh system heap, locks the location of this memory space, and then publishes a pointer to it by writing the address of the pointer to disk. Because LabVIEW allows the user access to primitive peak and poke memory functions, as well as the ability to link external code resources into VIs, we were able to write a VI to read the address pointer from disk and then poke data out into the structure at this address in memory.

Limitations of the present implementation of this system include the following factors. Although LabVIEW allows the inclusion of $\mathrm{C}$ code into instruments, it does not provide direct access to the Macintosh toolbox. Because of this, the programmer must go beyond the LabVIEW environment to produce features such as userdefinable windows for data. A second, related problem is that the interface to front-panel controls is not public, so that it is impossible to add user-written devices onto the menu of front-panel indicators and controls.

In all, however, we have found our experience with LabVIEW and the Macintosh to be extremely rewarding. The system is relatively cheap (relative, that is, to commercial "turnkey" systems). A suitable EEG machine is the major expense, at around $\$ 30,000$. A suitably equipped Mac IIfx is around $\$ 8,000$; LabVIEW is $\$ 1,995$, and the NuBus boards are around $\$ 1,500$ each. In many ways more important than this cost saving is the fashion in which this system encourages experimentation. The level of flexibility provided is absent in even the most expensive commercial systems, which provide only simple "form-based" control templates for the basic oddball, auditory EP, somatosensory EP, and other paradigms-a possible exception to this rule being the Nicolet pathfinder, which allows the user to program in Fortran 77 with full control over the Unix-based pathfinder. This Macintoshbased system implements many of the features of topographic mapping systems that cost much more, and it is completely programmable by members of the lab staff who wish to extend its capabilities.

\section{REFERENCES}

Agerwala, T., ARvind (1982). Data flow systems. IEEE Computer, $15,10-13$.

Adler, L. E., Pachtman, E., Franks, R. D., Pecevich, M., Waldo, M., Freedman, R. (1982). Neurophysiological evidence for a defect in neuronal mechanisms involved in sensory gating in schizophrenia. Biological Psychiatry, 17, 639-654.

Buchsbaum, M. S., a Silverman, J. (1968). Stimulus intensity control and the cortical evoked response. Psychosomatic Medicine, 30 , 12-22.

Buchsbaum, M. S., Haier, R. J., Johnson, J. (1983). Individual differences in augmenting/reducing evoked potentials. In A. Gale \& J. A. Edwards (Eds.), Physiological correlates of human behavior (Vol. 3, pp. 117-138). London: Academic Press.

MARK, D., REED, C. (1989). Macintosh programming primer inside the toolbox using Think's Lightspeed C. New York: Addison-Wesley. SHU, N. C. (1988). Visual programming. New York: Van Nostrand Reinhold.

\section{NOTES}

1. EEG machine advertisements may be found in the journal Electroencephalography \& Clinical Neurophysiology.

2. As of October, 1990, the most suitable Macintosh platform available is the IIfx model, which has 5 slots free to contain the boards necessary for full functionality. For stable operation, the program requires system software version 6.05 or better and, preferably, $8 \mathrm{MB}$ of memory. A large-screen gray-scale monitor is helpful in the use of LabVIEW, and a color or gray-scale monitor is essential for use of the Mapper program. Relevant cards installed in the Macintosh are the NB-MIO-16-9 16-channel A/D board or its successor the NB-MIO-16X-18, a 16-bit resolution model with enhanced features, and the NB-DMA-2800 block mode DMA and GPIB board.

(Manuscript received March 19, 1990; revision accepted for publication March 25, 1991.) 